\title{
Diacronie
}

Studi di Storia Contemporanea

$N^{\circ} 7,3 \mid 2011$

«Spagna Anno Zero»: la guerra come soluzione

\section{Milieux et mouvements libertaires pendant la Seconde République et la Guerre Civile espagnole}

\section{Arnaud Dolidier}

\section{(2) OpenEdition}

\section{Journals}

Édition électronique

URL : http://journals.openedition.org/diacronie/3225

DOI : 10.4000/diacronie.3225

ISSN : 2038-0925

Éditeur

Association culturelle Diacronie

Référence électronique

Arnaud Dolidier, « Milieux et mouvements libertaires pendant la Seconde République et la Guerre Civile espagnole », Diacronie [En ligne], N 7, 3 | 2011, document 5, mis en ligne le 29 juillet 2011, consulté le 06 mai 2019. URL : http://journals.openedition.org/diacronie/3225 ; DOI : 10.4000/diacronie.3225 


\section{Diacronie}

\section{Milieux et mouvements libertaires pendant la Seconde République et la Guerre Civile espagnole}

\section{Arnaud DOLIDIER *}

L'anarchisme est l'un des courants politique qui se distingue par sa diversité et son hétérogénéité. Pourtant, les milieux libertaires ont souvent été appréhendés par l'historiographie de façon homogène, contribuant à réifier leurs histoires. A rebours de ces écueils mais aussi des hagiographies militantes, l'objet de cet article est de présenter une synthèse des nouvelles interprétations sur l' "anarchisme" pendant la Seconde République et la Guerre Civile espagnole. On ne peut comprendre l'histoire des années trente en Espagne sans se pencher sur les discours et pratiques des milieux libertaires.

\section{Introducion}

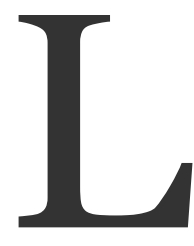

'histoire des milieux et des mouvements libertaires fut d'abord écrite par les militants, conscients du potentiel mobilisateur du discours historique ${ }^{1}$. L'anarchie s'est servie et se sert encore de l'histoire comme arme politique, pour diffuser des représentations homogènes basées sur une série de mythes. Ceux-ci permettent d'alimenter une identité collective essentialisée et sacralisée. Ainsi par exemple, des travaux comme ceux de José Peirats, Max Nettlau,

\footnotetext{
1 TAVERA, Susanna, «La historia del anarquismo español : una encrucijada
} interpretativa nueva», in Ayer, $\mathrm{N}^{\circ}$ 45, 2002, p. 19. 
Gaston Leval ou encore Abad de Santillan², sont des livres d'histoire anarchiste écrits par des militants à destination d'autres militants et sympathisants. Ils servent à justifier et légitimer des prises de positions politiques, des pratiques, notamment pendant la Guerre Civile espagnole. Ces ouvrages s'inscrivent dans le combat politique contre la dictature franquiste. Ils constituent un prolongement de la guerre.

Pendant la transition démocratique, l'histoire sociale a tenté de comprendre l'apparition et l'hégémonie de l'anarchisme espagnol au sein du monde ouvrier. Les thématiques portent principalement sur le développement des organisations anarchosyndicalistes à l'échelle régionale, leur rôle dans les mobilisations rurales, ainsi que pendant la Guerre de 1936. Des travaux se sont penchés sur les luttes sociales dans les campagnes au $\mathrm{XIX}^{\mathrm{e}}$ siècle, sur le phénomène du terrorisme début $\mathrm{XX}^{\mathrm{e}}$, ou encore sur l'évolution de l'idéologie politique libertaire ${ }^{3}$. Plusieurs historiens ont expliqué le phénomène de l'anarchisme comme mouvement millénariste s'accordant avec les modes de vie des communautés rurales. D’autres historiens ont réfuté cette thèse, soulignant au contraire que les mouvements libertaires étaient "rationnels", basés sur une solide "conscience de classe" 4 .

C'est pourtant sur la période des années trente - Seconde République mais surtout Guerre d'Espagne - que les historiens ont concentré leurs recherches ${ }^{5}$. Dans certains

$2 \quad$ PEIRATS, José, La CNT en la revolución española, 3 vols., Toulouse, Éditions CNT, 1952-1953 ; NETTLAU, Max, Esbozo de historias de las utopias, 1934; LEVAL, Gaston, La obra constructiva de la revolución española, México, Ed. Ideas, 1982; ABAD DE SANTILLAN, Diego, Por qué perdimos la guerra, Madrid, G del Toro, 1975.

3 De manière non exhaustive, sur l'anarchisme et le monde rural, on peut citer les travaux de MAURICE, Jacques, El anarquismo andaluz. Campesinos y sindicalistas. 18681936, Barcelona, Critica, 1990; BARRIO ALONSO, Ángeles, Anarquismo y anarcosindicalismo en Asturias (1890-1936), Madrid, Siglo XXI, 1988. Sur le terrorisme anarchiste, voir les travaux de LIDA, Clara Eugenia, La Mano Negra (anarquismo agrario en Andalucía), Viscaya, Ed. Zero, 1972, et, Anarquismo y revolución en la España del XIX, Madrid, Siglo XXI, 1972. Sur l'évolution de l'idéologie libertaire, voir ALVAREZ JUNCO, José, La ideología política del anarquismo español (1868-1910), Madrid, Siglo XXI, 1976.

4 L'anarchisme comme mouvement millénariste est développée dans les travaux de BERNALDO DE QUIROS, Constancio, el espartaquismo agrario andaluz, Madrid, Turner, 1974; dans celui de DIAZ DEL MORAL, Juan, Historia de las agitaciones campesinas andaluzas, Madrid, $2^{\mathrm{e}}$ ed, Alianza Ed., 1969 ; dans l'ouvrage de BRENAN, Gerald, The Spanish Labyrinth. An account of the social and political Background of the Civil War, Cambridge, University Press, 1944 et HOBSBAWM, Eric, Primitives Rebels. Studies in Archaic Forms of Social Movement in the $19^{\text {th }}$ and $20^{\text {th }}$ Centuries, Manchester, University Press, 1959. Cette thèse fut réfutée par les travaux de KAPLAN, Temma, Origenes sociales del anarquismo en Andalucia. Capitalismo agrario y lucha de clases en la provincia de Cadiz, 1868-1903, Barcelona, Crítica, 1977. Pour une synthèse de ce débat, voir BERNECKER, Walther, « Accion directa y violencia en el anarquismo espanol », in Ayer, $\mathrm{N}^{\circ}$ 13, 1994, pp. 147-188.

$5 \quad$ Sur les années trente, voir BERNECKER, Walter, Colectividades y revolucion social. El anarquismo en la Guerra Civil española, 1936-1939, Barcelona, Critica, 1982 ; BRADEMAS, John, Revolution and social revolution. A Contribution to the History of the Anarchosyndicalist Movement in Spain, thèse doctorale, Oxford, 1953 ; CASANOVA, Julián, De la calle al frente. El anarcosincalismo en Espana (1931-1939), Barcelona, Crítica, 1997 ; GUTIERREZ 
travaux, l'anarchisme espagnol est appréhendé grâce à un discours uniformisant, produit par les organisations de l'époque. Dans plusieurs récits, les protagonistes passent au second plan, l'analyse de "l'anarchisme" s'effectuant au détriment "des anarchistes". La culture politique libertaire est analysée comme un bloc, une « chose » homogène ${ }^{6}$. Par ailleurs, certains auteurs ont mis l'accent sur les responsabilités et les erreurs des organisations anarcho-syndicalistes pendant la guerre de 1936. Ils ont véhiculé des jugements de valeurs sur ceux et celles qui se revendiquaient de l'anarchie, leur attribuant au mieux une certaine naïveté quant à l'appréhension du contexte politique et social de l'époque, au pire la responsabilité du "désordre" dans le camp républicain7. Ces écueils révèlent une démarche téléologique, dans la mesure où l'historiographie s'est penchée sur l'histoire de "l'anarchisme" pour expliquer son rôle pendant la guerre de 1936. Ils révèlent également le fait qu'en étudiant des "structures" et des "organisations" sans interroger les agents historiques et leurs vocabulaires politiques, l'historiographie a produit des discours naturalisés et réifiés, prolongeant les luttes d'alors au lieu de les comprendre ${ }^{8}$.

L'historien Joaquin Romero Maura9 a fait remarquer que "l'anarchisme espagnol" n'était pas l'unique forme avec laquelle les acteurs de l'époque auraient pu interpréter le contexte social et politique. Ainsi, l'historicisation des vocabulaires politiques de l'époque et la déconstruction des identités collectives paraissent nécessaires pour comprendre comment les personnes interprètent leur réalité. Cette méthode conduit à la formulation de nouvelles problématiques : quelles sont les interactions entre les communautés rurales et urbaines traditionnelles et les secteurs anarchistes? Quels sont les phénomènes qui ont contribué à accroître un sentiment d'appartenance aux réseaux

MOLINA, José Luis, La idea revolucionaria. El anarquismo organizado en andalucía y Cadiz durante los años treinta, Madrid, Madre tierra, 1993.

$6 \quad$ La thématique de l'hétérogénéité et diversité de "l'anarchisme" est traitée dans BARRIO ALONSO, Ángeles, «El anarquismo asturiano. Entre el sindicalismo y la política, 1890-1920», in Ayer, 2002, op.cit., pp. 147-170.

$7 \quad$ C'est le cas de l'historien Javier Tusell : « En théorie il aurait suffit que la République utilise ses ressources, spécialement l'aviation et la marine, de manière cohérente et ordonnée, afin d'empêcher que Franco ne puisse traverser le détroit. Mais elle aurait pu le faire seulement s'il ne s'était pas produit une révolution », citation tirée de l'ouvrage de CRUZ, Rafael, En el nombre del pueblo. República, rebelión y guerra en la España de 1936, Madrid, Siglo XXI, 2006, p.241. La traduction est de nous.

8 Pour une critique historiographique de la guerre civile espagnole, voir SÁNCHEZ LEÓN, Pablo et IZQUIERDO MARTIN, Jesús, La guerra que nos han contado. 1936 y nosotros, Madrid, Alianza, 2006. Notamment le chapitre 3 de la première partie sur la naturalisation et le positivisme, pp. 87-140.

9 ROMERO MAURA, Joaquín, «El anarquismo : el caso espanol», in, ROMERO MAURA, Joaquín, La romana del diablo. Ensayos sobre la violencia política en España (1900-1950), Madrid, Marcial Pons, 2000, p. 89. 
libertaires ? Comment des milieux libertaires, qui ont toujours résisté à s'intégrer au système politique, participent au pouvoir au printemps 1936 puis en avril 1938 ? 


\section{Anarcho-syndicalisme et anarchisme pendant la Seconde République espagnole}

La Confédération Nationale du Travail (Confederación Nacional del Trabajo) naît en 1910 des compromis politiques entre différents groupes, collectifs et forces sociales, synthétisés dans la notion de communisme libertaire qu'il s'agit d'instaurer selon les principes de l'anarcho-syndicalisme. L'instabilité idéologique et politique de l'organisation se fait sentir très tôt lorsqu'elle conclut une alliance avec l'Union Générale des Travailleurs (Unión General de Trabajadores) en 1917, ou qu'elle adhère à la troisième internationale en 1922. Elle apparaît également sous la dictature de Primo de Rivera (1923-1930), lorsqu'une partie de l'organisation se montre favorable à une alliance avec les forces républicaines pour faire tomber le dictateur. C'est face à l'hétérogénéité des militants qui composaient la CNT que la Fédération Anarchiste Ibérique (Federación Anarquista Ibérica) naît en 1927, avec pour mission de combattre le "réformisme" et de défendre "la pureté" des principes de Bakounine.

Avec l'avènement de la Seconde République le 14 avril 1931, les organisations libertaires cessent d'être clandestines, et peuvent profiter de nouveaux espaces syndicaux et politiques. Cela se traduit par une augmentation sans précèdent de leurs effectifs. A l'automne 1931, la CNT compte 800.000 affiliés, dont 300.000 en Andalousie ainsi qu'en Catalogne ${ }^{10}$. En septembre et octobre 1931, de nombreuses occupations de fermes, grèves et manifestations sont menées par les habitants des campagnes afin de réclamer des augmentations de salaires, de meilleures conditions de travail, ainsi que l'embauche des journaliers condamnés au chômage. Des réformes dans le monde du travail et dans les campagnes sont élaborées par la République pour tenter de répondre à des problèmes anciens, comme le chômage ou l'accès à la terre. Elles s'accompagnent de l'effort des syndicats socialistes, UGT et Fédération Nationale des Travailleurs de la Terre (Federación Nacional de Trabajadores de la Tierra), pour contraindre les propriétaires et les patrons réticents à appliquer ces réformes. La CNT quant à elle, refuse d'accorder une légitimité au nouveau régime politique. La conception de la grève, tout comme d'autres formes d'actions collectives, signifie pour la plupart des militants libertaires un préambule à la "révolution"11. Ainsi, la stratégie

\footnotetext{
$10 \quad$ CASANOVA, Julián, op.cit., p. 28.

$11 \quad$ Ibidem, p. 49.
} 
de l'action directe, conçue comme le refus de tout arbitrage de l'État et de toute négociation, se heurte à l'instauration des Jurados Mixtos ${ }^{12}$.

Les milieux anarchistes tentent de transformer et d'interpréter chaque geste d’indiscipline, de délit, comme un acte révolutionnaire porté contre le pouvoir ${ }^{13}$. Les expériences de luttes sociales sont le terreau permettant aux organisations révolutionnaires de diffuser des vocabulaires politiques. L'augmentation des conflits s'accompagnent d'une diffusion de termes re-signifiés, en rupture avec les catégories républicaines. Les expériences de luttes sociales, ainsi que les multiples espaces d'échanges politiques comme les athénées libertaires, rendent possible la construction de "pratiques discursives". De même, bien qu'elles se soldent par des échecs sanglants, les trois tentatives insurrectionnelles déclenchées en l'espace de deux ans par des militants anarchistes ${ }^{14}$, sont l'objet d'interprétations spécifiques permettant de renforcer la cohésion du groupe.

En effet, l'envoi systématique de corps militarisés pour garantir le maintien de l'ordre public conduit à des massacres qui, au lieu de faire taire la contestation, lui donnent de nouveaux motifs pour se poursuivre. L'action qui consiste à tuer ou à envoyer en prison les militants anarchistes les plus violents s'accompagne de discours qui mettent l'accent sur l'indignation, la compassion, et qui participent à construire de nouvelles vagues de protestations ${ }^{15}$. Les campagnes pro-presos initiées par la FAI sont le résultat de pratiques solidaires nées de l'expérience douloureuse de la répression. Elles permettent de dynamiser le militantisme ; la répression permet d'accentuer la cohérence et l'unité de l'identité collective. C'est dans ce cadre qu'il faut appréhender les journées de protestation contre la répression, contre la loi de Défense de la République $^{16}$, contre les déportations de militants suite aux insurrections ${ }^{17}$. A partir de

12 Commissions paritaires instaurées pour régler les conflits entre patrons et ouvriers sous l'arbitrage de l'État à partir de 1932. Ces instances, aussi bien dans le monde rural que urbain, freinaient les tactiques d'action directe de la CNT obligée d'agir en dehors de la nouvelle légalité. Elles ont permis dans le même temps d'aider l'UGT et la FNTT à accroître leur stratégie syndicale.

13 ROMERO MAURA, Joaquín, op.cit., p. 97.

14 Les secteurs partisans d'une rupture radicale avec le système politique proposèrent de suivre la voie insurrectionnelle. Des tensions internes apparurent au sein des organisations libertaires. Face à un courant "réformiste" nommé les "trentistes", partisans de l'intégration au système républicain, les défenseurs de l'insurrection provenant principalement de la FAI imposèrent leurs stratégies, conduisant à l'exclusion de nombreux anarcho-syndicalistes tels que Angel Pestaña ou Joan Peiro. Sur la stratégie insurrectionnelle, voir UCELAY-DA CAL, Enric, TAVERA, Susanna, « Una revolución dentro de otra : la logica insurrectional en la política española, 1924-1934 ", in Ayer, 1994, op.cit., pp. 115-146.

15 ROMERO MAURA, Joaquín, op.cit., pp. 102-103.

16 Loi votée par les Cortes en octobre 1931. Elle constitue le volet répressif de la République, permettant de suspendre les droits politiques, fermer les locaux syndicaux, 
1932, le registre émotionnel, la diffusion de discours contre la répression et de slogans tels que « España entera es una carcel », définissent les politiques d’identification de la CNT et de la FAI.

Le régime de la Seconde République est dépositaire d'une conception militarisée de l'ordre public. Patrick Bruneteaux définit le concept d'ordre public comme «l'intégration de la violence sociale dans un système institutionnel légitime ${ }^{18}$. L'ordre public est un concept restrictif né du besoin des classes dominantes de délégitimer toute forme de violence politique et sociale. Restrictif puisque chaque mobilisation collective est interprétée par les dirigeants politiques comme un danger potentiel pour le régime. Ce danger, au lieu d'être canalisé et maîtrisé par l'Etat, fait l'objet de pratiques destructrices par les instances militaires. "L'ordre public" consiste à garantir l'expression de droits politiques subordonnés au contrôle militaire, de punir des forces politiques dissidentes au lieu de les intégrer institutionnellement à l'ordre républicainn ${ }^{19}$.

Après de nombreux conflits et trois insurrections manquées, les organisations libertaires connaissent une période de reflux. A l'échelle nationale, les chiffres sont éloquents : des 800 ooo adhérents durant 1931, il n'en reste plus que 300000 après $1933^{20}$. Les conflits internes ainsi que l'échec des tentatives insurrectionnelles portent une grande responsabilité dans la faiblesse numérique des organisations libertaires. Cependant, la fermeture des locaux syndicaux, des centres culturels, la censure des journaux anarchistes, expliquent cette baisse des effectifs qui ne signifie pas pour autant que le nombre de personnes se revendiquant "anarchistes" ait diminué.

En mars 1934, une longue grève à Saragosse dans le secteur des transports publics est appelée conjointement par l'UGT et la CNT. La répression donne lieu à de nouvelles mobilisations pour libérer les militants emprisonnés et réembaucher les travailleurs licenciés ${ }^{21}$. Ainsi, à partir de 1934, la CNT convoque des grèves "défensives" pour améliorer la condition ouvrière tout en continuant à diffuser un discours "révolutionnaire". De même, ce fait marque le début d'une possible alliance entre les

interdire les manifestations et censurer des journaux. Elle est remplacée par la loi d'Ordre Public en juillet 1933, davantage répressive.

$17 \quad$ L'un des faits marquant pendant ces trois épisodes insurrectionnels fut l'événement de Casas Viejas en janvier 1933. Dans ce village de la province de Cadix, des paysans prirent le contrôle du pouvoir local avant de proclamer le communisme libertaire. Une fois sur place, l'armée tua les meneurs de l’insurrection. Sur les événements de Casas Viejas, voir MINTZ, Jérome, Los anarquistas de Casas Viejas, Granada, Diputación de Granada, 1999.

18 BRUNETEAUX, Patrick, Maintenir l'ordre, Paris, Presse de Sciences Politiques, 1996, p. 34, cité in, GODICHEAU, François, La Guerre d'Espagne. République et Révolution en Catalogne (1936-1939), Paris, Odile Jacob, 2004, p. 18.

19 Pour comprendre le passage en France de la répression militaire au maintien de l'ordre assuré par les forces de polices, voir BRUNETEAUX, Patrick, Maintenir l'ordre, cit.

$20 \quad$ CASANOVA, Julián, op.cit., p. 84.

$21 \quad$ Ibidem, p. 134. 
deux centrales syndicales. Bien que les journaux de l'époque laissent paraitre une cohésion sans faille, il ne fait aucun doute qu'entre ces nouvelles mobilisations et les tenants d'un "pur" discours révolutionnaire, les milieux libertaires sont traversés par une palette d'opinions et d'interprétations diverses. De même, la possibilité d'alliance avec l'UGT fait débats, beaucoup considérant que celle-ci a sa part de responsabilité dans les massacres antérieurs.

La CNT ne prend pas part à l'insurrection d'octobre 1934 compte tenu de sa faiblesse numérique, des stigmates de la répression antérieure et des réticences de certains militants à s'allier à l'UGT. Seule la section des Asturies participe à la révolte. Cependant, cet événement s'accompagne d'une multitude de commentaires de la part des dirigeants et des partis politiques, permettant de construire, inventer, sélectionner, définir l'affrontement selon des significations précises ${ }^{22}$. La plus éloquente est le parallèle opéré entre cette insurrection et la révolution russe d'octobre 1917, permettant de diffuser l'idée selon laquelle les forces syndicales, politiques et associatives de la gauche sont aux mains de Moscou. Cela s'accompagne également de l'exclusion politique $^{23}$ de tous ceux qui représentaient la "classe", mais aussi la "communauté populaire”. Octobre 1934 est un événement fondateur compte tenu de l'inflation des interprétations par les groupes de l'époque. Il permet de dessiner deux communautés de sens distincts, chacune d'entre elles revendiquant la représentation d'un peuple "catholique" ou "travailleur" antagonique l'une de l'autre, signifiant l'exclusion politique de l'adversaire ${ }^{24}$. Ce processus d'invention d'octobre connaît son apogée pendant la bataille électorale de février 1936, remportée par la coalition du Front Populaire.

\section{Le printemps 1936}

L’image fréquemment répandue sur le printemps 1936 est celle d'une période où une vague de grève sans précédent déferle sur l'Espagne, contre un gouvernement incapable de faire face à une situation de désordre public. Cette vision a constitué un mythe qui a permis de diffuser l'idée qu'une guerre civile était inévitable ${ }^{25}$. Le gouvernement, en dépit de ses discours progressistes, devient hostile au mouvement social. Loin d'être dépassé par la situation, il continue à pratiquer la pénalisation des

$22 \quad$ CRUZ, Rafael, op.cit., pp. 70-75.

23 Les travailleurs affiliés à des syndicats sont licenciés, les journaux sont censurés, de nombreux locaux syndicaux et culturels sont mis sous scellés, etc.

$24 \quad$ Ibidem, p. 74.

$25 \quad$ Ibidem, pp. $180-188$. 
opinions, l'interdiction arbitraire des manifestations et la suspension des garanties constitutionnelle jusqu'en juillet 1936. L'un des éléments qui permet d'expliquer la construction imagée du printemps 1936 comme période révolutionnaire instable n'est pas l'imminence d'une révolution sociale, mais bien la visibilité croissante de la violence politique dans les grands pôles urbains à partir d'avril $1936^{26}$. Tous les faits violents, aussi minimes furent-ils, sont magnifiés et politisés par chacune des communautés politiques. Toutefois, les violences urbaines étaient fréquentes. Des heurts entre militants ont lieu dans des zones urbaines telles que Valence, Séville ou Barcelone, mais la situation est loin de ressembler au "chaos" décrit par les militaires rebelles.

Par ailleurs, la plupart des mouvements sociaux de cette période se font sur la base de revendications matérielles adressées au gouvernement du Front Populaire. Ainsi par exemple, la Fédération Espagnole des Travailleurs de la Terre (Federación Española de Trabajadores de la Tierra) ${ }^{27}$ organise des occupations massives de fermes afin que le gouvernement accélère ses réformes agraires. Quant aux secteurs anarchistes, ils se trouvent en position de faiblesse, n'ayant pas les forces d'impulser des mobilisations sociales. Cependant, dans certains pôles urbains comme Málaga, ils parvinrent à convoquer quelques actions de contestation, bien éloignées de l'action directe. En effet, au lieu de mobiliser ses affiliés contre le régime, priorité est donnée à la négociation, sur des questions telles que le chômage ou les conditions de travail ${ }^{28}$.

Lors de son congrès en mai 1936 à Saragosse, la CNT reconnaît pour la première fois les erreurs de la stratégie insurrectionnelle. Les revendications concrètes sur les salaires ou les conditions de travail sont mises en avant contre les grèves "politiques", sans pour autant renoncer aux principes révolutionnaires. Les militants et les sections exclus de la CNT quelques années auparavant sont réintégrés. Ce congrès s'achève avec la publication d'un programme politique où le communisme libertaire comme finalité est réaffirmé, et dont les moyens pour l'atteindre est la création de communes libres et le développement de l'autogestion ${ }^{29}$. Mais le coup d'État de juillet 1936 surprend les réseaux de l'anarcho-syndicalisme espagnol qui pour la plupart sont en pleine restructuration.

26 GONZALEZ CALLEJA, Eduardo, «La dialéctica de las pistolas. La violencia y la fragmentación del poder político durante la Segunda República», in, MUÑOZ SORO, Javier, LEDESMA, José Luis, RODRIGO, Javier (coord.), Culturas y políticas de la violencia. España siglo XX, Madrid, Siete Mares, 2005, p. 140.

$27 \quad$ La FETT succède à la FNTT durant le second bienio (1934-1936).

28 CASANOVA, Julián, op.cit., p. 148.

29 BERNECKER, Walther, "Accion directa" y violencia en el anarquismo espanol», op.cit., p. 188. 


\section{Révolution et contre-révolution}

Rafael Cruz a démontré30, à rebours des mythes couramment diffusés par l'historiographie hispaniste et espagnole, que ce n'est pas «le peuple en arme » qui vainc les militaires. L'échec du coup d'État doit être recherché dans l'affrontement entre les forces militaires rebelles et résistantes, en y incluant les corps de polices militarisées. Dans les villes où la garnison déclare l'état de guerre et est suivie par les forces de la Garde Civile, toute résistance est inutile. Dans celles où la garnison et les forces policières restent indécises, le contrôle républicain demeure intact. Enfin, là où la Garde Civile et la Garde d'Assaut combattent contre les rebelles, le soulèvement échoue ${ }^{31}$. Bien entendu, au côté des militaires restés fidèles à la République, les organisations ouvrières sont aussi présentes. Toutefois, seuls les militants les plus combatifs ainsi que quelques dirigeants sortent dans les rues au côté des forces de sécurité. Ce n'est que dans les jours qui suivent ces journées de juillet que les habitants envahissent les rues ${ }^{32}$. Armées par le pouvoir républicain, les organisations anarchosyndicalistes notamment, ont pu s'approprier la victoire à plusieurs endroits, les forces de l'ordre loyalistes renonçant à un affrontement.

L'attribution par le gouvernement d'armes aux organisations ouvrières n'est pas décisive pour contenir l'insurrection. Cependant, l'État perd le monopole de la violence et ne peut freiner dans les provinces le déclenchement d'un processus révolutionnaire. Les syndicats ouvriers profitent de l'opportunité politique de la rébellion militaire pour déployer le répertoire d'actions collectives insurrectionnelles urbaines et rurales ${ }^{33}$. Dans les provinces où le soulèvement militaire échoue, les organes de l'État républicain se retrouvent dans l'incapacité d'exercer un quelconque pouvoir : les gouverneurs civils restent à la tête d'une administration fantôme. Quant au pouvoir local lié aux municipalités, partout il est supplanté par des comités étiquetés "révolutionnaires" ou de "défense". Ces comités, construits et dirigés par les syndicats ouvriers, prennent le contrôle de la mobilisation économique, politique et militaire, sans détruire les

\footnotetext{
3o $\quad$ CRUZ Rafael, op.cit., p. 238.

Ibidem, p. 239.

Ibidem.

Ibidem, p. 242. Sur le concept de répertoire d'actions collectives, voir TILLY, Charles, «Les origines du répertoire de l'action collective contemporaine en France et en GrandeBretagne », in Vingtième Siècle. Revue d'histoire, N 4, octobre 1984, pp. 89-108.
} 
anciennes municipalités, qui dans la plupart du temps continuent de siéger à vide ${ }^{34}$. Des situations de souverainetés multiples détrônent le pouvoir étatique sans le conquérir. Dans les grandes villes, des comités antifascistes prennent la direction du mouvement révolutionnaire. C'est le cas de pôles urbains comme Barcelone, Málaga, Valence, Aliquante, etc. Toutefois, aucune force organisationnelle n'est capable d'instaurer une domination politique sur les territoires ayant échappé aux rebelles.

Durant les premières semaines du conflit, la prise de conscience qu'il s'agit d'une guerre ainsi que l'identification des camps et des forces politiques est inintelligible, confuse, contrairement à ce que diffusent ensuite les propagandes officielles de chaque camp35. Les organisations libertaires, protagonistes dans la résistance face aux rebelles, intensifient leurs discours de propagande pour diffuser une interprétation homogène de la situation. En ce début de guerre, les vocabulaires politiques acquièrent une dimension performative. "Dire" équivaut à "faire". Ainsi, les réseaux libertaires sont les premiers à attribuer une signification particulière à un conflit qui n'a pas encore de nom. Ils proclament la révolution, de sorte que leurs discours se diffusent largement. Un journal comme «Solidaridad Obrera » en Catalogne tire 31.00o exemplaires au début du mois de juillet. Fin août, son tirage s'élève à plus de 150.000 exemplaires ${ }^{36}$.

Les organisations libertaires, dans un premier temps, ne sont pas tant préoccupées à collectiviser les moyens de production qu'à combattre leurs ennemis politiques. La presse exhorte les habitants à "purifier" la révolution. Propriétaires, techniciens, directeurs, droitiers sont assassinés. De même, l'anticléricalisme s'exprime avec virulence : incendie d'objets et d'images du culte religieux, assassinats de membres du clergé, exhumation de corps de moines et de bonnes sœurs, etc. L'action combinée des milices et des comités débouche sur une sanglante épuration ${ }^{37}$. Les multiples figures que revêt "l'ennemi de classe" doivent être combattues. Les libertaires incitent les personnes à éliminer tous les symboles du pouvoir, qu'ils soient militaires, économiques, culturels ou ecclésiastiques. Ces violences, couramment qualifiées d' "irrationnelles" et d' "incompréhensibles" par l'historiographie, sont le fruit d'une

34 GODICHEAU, François, "Frankenstein républicain : l'évolution autoritaire de la République espagnole en guerre », in, La Seconde République espagnole, Pau, Editions Cairn, 2008, pp. 49-75, p. 53.

35 GODICHEAU, François, "Guerre Civile", "Révolution", "Répétition Générale" : les aspects de la Guerre d'Espagne ", in, BOURDERON, Roger (dir.), La Guerre d'Espagne : l'histoire, les lendemains, la mémoire, Paris, Tallandier, 2007, pp. 89-105, p. 93.

$36 \quad$ CASANOVA, Julián, op.cit., p. 165.

$37 \quad$ Bien entendu, les milieux libertaires ne furent pas les seuls à appeler les habitants à se débarrasser des "ennemis de la révolution". Sur les exactions, les assassinats et les multiples formes de répression pendant la guerre, voir l'ouvrage de SANTOS, Juliá (coord.), Víctimas de la Guerra Civil, Madrid, Temas de Hoy, 1999. 
recomposition des identités politiques à partir d'éléments déjà existants, qui dessinent de nouvelles frontières entre ennemis et amis. L'irruption brutale du phénomène de la violence politique peut être appréhendée grâce à ce qu'Alessandro Pizzorno appelle des moments de "politique absolue" 38 où les mots acquièrent une signification exclusive. La peur d'un danger et d'une menace sur le groupe, sur ses catégories et ses référents, contribue à transformer l'adversaire d'hier en ennemi d'aujourd'hui. Ainsi, la violence politique doit être perçue par l'observateur contemporain comme la conséquence d'un processus de nouvelles "pratiques discursives" permettant aux personnes d'appréhender une situation inédite. Comme l'ont fait remarquer Pablo Sánchez León et Jesús Izquierdo Martín dans un récent ouvrage, « le sujet avait la sensation que s’il n'exprimait pas par des actes son identification avec ses référents, il cesserait lui-même d'être celui qu'il était, d'exister au sens moral»39.

"Dire et Faire la révolution" pour les organisations libertaires se traduit par une inversion des codes culturels que l'on aperçoit dans des villes comme Barcelone, Valence ou Málaga. L’instauration de symboles bientôt hégémoniques est le signe de "l'avènement de la révolution". Au départ, son existence n'est pas tant liée à un ensemble de faits et de mobilisations qu'à sa proclamation par les organisations ellesmêmes. Elles sont rapidement dépassées par le "pouvoir d'agir" des protagonistes, chacun agissant selon son ressenti du phénomène "Révolution". Ainsi par exemple, déclenchée par les organisations ouvrières, la grève se retrouve très vite hors de contrôle des comités syndicaux. Lorsque les consignes signalent sa fin, les ouvriers refusent de reprendre le travail, "révolution" signifiant pour beaucoup fin de "l'exploitation salariale"40. De même, le processus de collectivisation s'impose très vite dans de nombreux secteurs tels que les industries, commerces, transports publics, café et salons de coiffure. La collectivisation des terres est menée dans les exploitations abandonnées par leurs propriétaires, ou dans les fermes directement saisies par les comités révolutionnaires. Cependant, si des journaliers sans terre et quelques propriétaires pauvres se lancent dans la collectivisation, bien des villages et des secteurs sont réticents à ce projet. Une situation hétérogène conduit le projet de collectivisation à s'imposer directement dans certains endroits, plus lentement dans

38 PIZZORNO, Alessandro, Le radici della politica assoluta e altri saggi, Milano, Feltrinelli, 1993.

39 IZQUIERDO MARTÍN, Jesús, SÁNCHEZ LEÓN, Pablo, op.cit., p. 270. La citation traduite est de GODICHEAU, François, "L'événement et les catégories du social », in, RIVALAND-GUÉGO, Christine, RODRIGUES, Denis, Écho de l'événement de l'époque moderne à l'époque contemporaine, Rennes, PUR, à paraître en 2011, p. 18.

$40 \quad$ CRUZ, Rafael, op.cit., p. 244. 
d'autres ${ }^{41}$. En effet, en Catalogne mais aussi en Andalousie, en Castille et dans le Levant, les petits exploitants et les métayers sont réticents à la collectivisation des terres. Certains l'acceptent, d'autres préfèrent sauvegarder leurs petite propriété. C'est le cas de villages comme Amposta en Catalogne ${ }^{42}$.

Pour ses partisans comme pour ses opposants, la révolution est une réalité tangible impossible à nier. Pendant l'été 1936, les dirigeants emploient le langage de la révolution, non sans ambiguïté et polysémie afin de gagner du temps et freiner le processus révolutionnaire. Rapidement, les adversaires de la révolution sociale diffusent l'idée que les institutions républicaines sont les seules forces capables de mener la guerre contre ses ennemis. L’idée d'une essence républicaine garante des conquêtes révolutionnaires fait son chemin. Cela permet de reconstruire un État dont le caractère institutionnel est profondément modifié par le mouvement révolutionnaire ${ }^{43}$. En effet, lorsque se constitue le gouvernement de Largo Cabellero en septembre 1936, l'entrée des forces politiques révolutionnaires présentes physiquement sur le terrain, c'est-à-dire leur intégration institutionnelle, permet à l'État de s'adapter au nouveau contexte. Fondées à l'origine sur un discours garantissant la révolution, ces institutions dont la nature même est reformulée, peuvent de manière équivoque conjuguer une synthèse entre "révolution" et "ordre public". De sorte que "l'ordre public" se combine au thème de la guerre, celle-ci devenant primordial pour défendre la révolution. L'argument qui consiste à promouvoir la reconstruction de l'État au nom de la révolution glisse peu à peu vers la protection d'un ordre républicain re-signifié, menacé à la fois par les franquistes et par des "incontrôlés" de l'arrière républicain. L'ordre public s'érige contre "l'anarchie", contre la "désorganisation" d'une "cinquième colonne". La construction de nouvelles figures de l'altérité, et l'émergence de "l'unité antifasciste" teintée d'un langage patriotique, recouvre les significations révolutionnaires qui se sont imposées dans un premier temps pour nommer les événements.

\section{Une guerre civile dans la guerre civile}

${ }_{41}$ CASANOVA, Julián, op.cit., p. 174. Sur les collectivisations au début de la Guerre Civile espagnole, voir BERNECKER Walter, Colectividades y revolucion social. El anarquismo en la Guerra Civil española, 1936-1939, cit. Voir aussi MINTZ, Frank, Autogestion et anarchosyndicalisme, Paris, Editions CNT, 1999.

${ }_{42} \quad$ Voir l'ouvrage de DUPONT, Cédric, Ils ont osé ! Espagne 1936-1939, Toulouse, Los Solidarios, 2002. L'ouvrage contient de nombreux documents de l'époque qui éclaire sur les processus de construction des collectivités.

43 GODICHEAU, François, « Frankenstein républicain... », cit., pp. 64-66. 
La vision communément acceptée des conflits au sein du « camp républicain » est celle d'un clivage frontal entre organisations révolutionnaires et contrerévolutionnaires (Parti Ouvrier d'Unification Marxiste44, POUM, et CNT contre Parti Socialiste Unifié de Catalogne45, PSUC, et Parti Communiste Espagnol46, PCE). Pourtant, les lignes de fractures sont aussi horizontales. Les conflits entre direction cénétiste et militants radicaux sont une réalité. En effet, l'argument de la direction anarcho-syndicaliste pour justifier la délégation de ministres au sein du gouvernement en septembre 1936 est de dire que le cadre politique a changé, que les institutions ont acquis un caractère révolutionnaire. Dans le discours des dirigeants révolutionnaires, la révolution n'est plus considérée comme un processus en cours mais comme une réalité figée qu'il faut à présent sauvegarder, défendre, protéger. La révolution est en quelque sorte "congelée" 47 au nom de la guerre à mener. Ces discours mettent en évidence de nouvelles tensions au sein des réseaux libertaires ${ }^{48}$. Devant l'impossibilité des militants réfractaires à formuler des alternatives, le chemin proposé par les directions est accepté au nom du "réalisme" politique.

Cette intégration facilite la reconstruction de l'État républicain, non seulement à l'échelle nationale mais aussi locale. Ainsi par exemple, après la dissolution à Barcelone du Comité Central Des Milices Antifascistes, toutes les organisations présentes au sein du gouvernement de la Généralité de Catalogne signent à l'automne 1936 les décrets permettant de dissoudre les comités révolutionnaires et de réorganiser les municipalités. Le POUM et la CNT font pression sur leurs bases pour imposer ces décrets. Des travaux ont montré les résistances de ces comités en Catalogne face la réorganisation administrative de l'État49. Pendant l'hiver 1936 jusqu'en mai 1937, un affrontement interne oppose une partie des bases de la CNT, de la FAI et du POUM à ses dirigeants. Les multiples provocations policières et politiques déclenchent un affrontement direct entre révolutionnaires et anti-révolutionnaires dans les rues de Barcelone. Dans ce conflit, les directions du POUM et de la CNT-FAI restent en retrait,

\footnotetext{
$44 \quad$ Partido Obrero de Unificación Marxista.

45 Partido Socialista Unificat de Catalunya.

$46 \quad$ Partido Comunista de España.

$47 \quad$ CASANOVA, Julián, op.cit., p. 176.

48 Peu d'anarchistes se sont élevés contre l'entrée de quatre dirigeants de la CNT dans le gouvernement. Ce n'est que plus tard avec les conséquences de cette décision que plusieurs militants ont contesté cette démarche. Pour plus de détails, voir Ibidem, pp. 177-197 et LORENZO, César, Les anarchistes espagnols et le pouvoir, Paris, Ed. Seuil, 1969.

$49 \quad$ Sur la constitution des comités en Catalogne, leurs rôles et résistances, voir le travail POZO GONZÁLEZ, José Antonio, El poder revolucionari a Catalunya durant los mesos de juliol a octubre de 1936. Crisi y recomposició de l'Estat, thèse de doctorat, Universitat Autonoma de Barcelona, 2002.
} 
et font pression pour que les militants libertaires cessent le combat ${ }^{50}$. La CNT, purgée de ses éléments révolutionnaires (assassinés ou envoyés en prison) peut réintégrer le gouvernement du front populaire antifasciste en avril 1938, orienté vers un programme de "guerre de libération nationale". Ainsi, la défaite de mai 1937 achève l'intégration et l'homogénéisation des organisations "révolutionnaires" dans le "camp républicain", permettant la reconstruction d'un État profondément autoritaire reconstitué autour de son armée et de ses forces de sécurité, et fondée sur un discours de mobilisation nationale. La "domestication" de la CNT mais aussi de l'UGT, permis à l'Etat d'accroître l'effort de guerre. De nouvelles pratiques de contrôle et de gestion des populations achèvent une normalisation institutionnelle et politique. Dans ces dispositifs, la justice joue un rôle central. Elle devient l'une des pierres angulaires des techniques disciplinaires et coercitives de l'État. Le contexte de la guerre, le sens patriotique qu'on lui attribue, conduit à l'instauration d'un État fondé sur une législation d'exception.

\section{Conclusion}

Les hagiographies et les histoires "officielles" des organisations libertaires s'inscrivent dans les langages et les arguments de l'époque justifiant les choix opérés par leurs directions. Les histoires de la CNT et de la FAI n'ont pas été écrites par les militants de base, mais par les cadres et les dirigeants qui, une fois dans l'exil, n'ont eu de cesse de justifier leurs décisions. Concernant l'histoire académique, les explications se fondent encore sur les oppositions du passé par le biais de concepts et d'un vocabulaire produits pendant la guerre. Le mythe de l'objectivité51, les écueils du néopositivisme, cachent des "pratiques discursives" qui se distinguent par leur naturalisation ${ }^{2}$. La volonté de s'affranchir des luttes passées paraît indispensable pour comprendre les phénomènes, mobilisations et démobilisations des hommes et des femmes pendant la guerre. Cela permet à l'historien de construire ses interprétations

50 GODICHEAU, François, «Les aspects de la Guerre d'Espagne », cit., p. 104. Sur les journées de mai 1937, voir EALHAM, Chris, La lucha por Barcelona. Clase, cultura y conflicto (1898-1937), Madrid, Alianza, 2005. On pourra lire les témoignages de Diego Abad de Santillan, Juan Garcia Oliver in, A CONTRETEMPS: D'une Espagne rouge et noir, Paris, Les éditions libertaires, 2009.

$51 \quad$ Sur le mythe de l'objectivité dans l'historiographie hispaniste et espagnole, voir l'article de SÁNCHEZ LEÓN, Pablo, "La objetividad como ortodoxia: los historiadores y el conocimiento de la Guerra Civil española », in, AROSTEGUI, Julio, GODICHEAU, François (eds.), Guerra Civil : mito y memoria, Madrid, Marcial Pons, 2006, pp. 95-135.

$5^{2}$ SÁNCHEZ LEÓN, Pablo, IZQUIERDO MARTÍN, Jesús, La guerra que nos han contado. 1936 y nosotros, op.cit., pp. 106-118. 
au-delà des langages institutionnels et organisationnels de l'époque qui ne recoupent pas toujours les réalités historiques.

Ainsi, il n'existe pas "d'essence libertaire". Les identifications collectives et les pratiques sociales anarchistes évoluent constamment selon l'appréhension des contextes sociaux et politiques. Il ne s'agit pas d'une essence inscrite dans la nature ni même dans l'ordre social, mais bien de pratiques re-signifiées historiquement pour combattre des formes multiples de dominations. Les discours organisationnels doivent être déconstruits pour comprendre comment des cultures politiques ont évolué, notamment en période de guerre. 


\section{* L'auteur}

Arnaud Dolidier est doctorant à l'université Michel de Montaigne Bordeaux 3 avec une thèse intitulée: «Repartir à l'assaut du ciel! Les mouvements libertaires durant la transition démocratique espagnole: ordre public et actions collectives (1975-1982)», sous la direction de M. François Godicheau. Domaine de recherche: histoire contemporaine de l'Espagne. Il a réalisé les Master 1 et Master 2 entre 2008 et 2010 sous la direction de M. Jean François Soulet et M. François Godicheau à l'université Toulouse 2 Le Mirail sur le thème: «La construction des identités collectives dans le milieu rural andalou pendant la Seconde République». Il est membre de l'association «Adelante».

URL: < http://studistorici.com/progett/autori/\#Dolidier >

\section{Per citare questo articolo:}

DOLIDIER, Arnaud, «Milieux et mouvements libertaires pendant la Seconde République et la Guerre Civile espagnole», Diacronie. Studi di Storia Contemporanea: Spagna Anno Zero: la guerra come soluzione, 29/07/2011,

URL:<http://www.studistorici.com/2011/07/29/dolidier_numero_7>

Diacronie Studi di Storia Contemporanea $\sqrt{ }$ www.diacronie.it

Risorsa digitale indipendente a carattere storiografico. Uscita trimestrale.

redazione.diacronie@hotmail.it

Comitato di redazione: Marco Abram - Giampaolo Amodei - Jacopo Bassi - Luca Bufarale - Alessandro Cattunar - Alice De Rensis Barbara Galimberti - Deborah Paci - Fausto Pietrancosta - Martina Sanna - Matteo Tomasoni - Luca Zuccolo

Diritti: gli articoli di Diacronie. Studi di Storia Contemporanea sono pubblicati sotto licenza Creative Commons 2.5. Possono essere riprodotti a patto di non modificarne i contenuti e di non usarli per fini commerciali. La citazione di estratti è comunque sempre autorizzata, nei limiti previsti dalla legge. 